FIAN/TD-24/07

ITEP/TH-57/07

\title{
Seiberg-Witten Theory and Extended Toda Hierarchy
}

\author{
A. Marshakov \\ Theory Department, P.N.Lebedev Physics Institute, \\ Institute of Theoretical and Experimental Physics, \\ Moscow, Russia \\ e-mail: mars@lpi.ru, mars@itep.ru
}

\begin{abstract}
The quasiclassical solution to the extended Toda chain hierarchy, corresponding to the deformation of the simplest Seiberg-Witten theory by all descendants of the dual topological string model, is constructed explicitly in terms of the complex curve and generating differential. The first derivatives of prepotential or quasiclassical tau-function over the extra times, extending the Toda chain, are expressed through the multiple integrals of the Seiberg-Witten one-form. We derive the corresponding quasiclassical Virasoro constraints, discuss the functional formulation of the problem and propose generalization of the extended Toda hierarchy to the nonabelian theory.
\end{abstract}

\section{Introduction}

The appearance of integrable systems in the context of the Seiberg-Witten theory is now clearly related to the gauge/string duality. The quasiclassical tau-functions or the infrared prepotentials, which give the exact low-energy effective actions on the gauge side, become identified in this framework with generating functions of the particular topological string models on the string side of duality. For example, the simplest possible quasiclassical tau-function of extended Seiberg-Witten theory explicitly coincides $[1,2]$ with the "half-truncated" generating function for the Gromov-Witten classes on $\mathbb{P}^{1}$ or the correlation functions of the topological $\mathbb{P}^{1}$ string model.

The gauge/string vocabulary looks here as follows: we compare the oversimplified but perturbed in the ultraviolet, simplest possible " $U(1)$ " Seiberg-Witten theory (to be seen, for example, as naive $N_{c}=1$ particular case of the $U\left(N_{c}\right)$-family) with the topological string model, describing quantum cohomologies of $\mathbb{P}^{1}$, to be generally identified with the base curve of the 
asymptotically free Seiberg-Witten theory. The variable $a$, coupled to the unity operator $\mathbf{1}$ of string theory, is identified with the only condensate $\langle\phi\rangle=a$ on the gauge theory side, while the variable $t_{1}=\tau_{0}=\frac{\vartheta_{0}}{2 \pi}+\frac{4 \pi i}{g_{0}^{2}}$, coupled to the Kähler class $\varpi$ of $\mathbb{P}^{1}$ target space, is identified with the (complexified) coupling constant. Moreover, it turns out, that all perturbations of the gauge theory, encoded in the ultraviolet prepotential

$$
F_{U V}(x ; \mathbf{t})=\mathbf{t}(x)=\sum_{k>0} t_{k} \frac{x^{k+1}}{k+1}
$$

correspond to switching on all gravitational descendants $\oplus_{k>0} t_{k+1} \sigma_{k}(\varpi)$ of the Kähler class $\varpi$ of the $\mathbb{P}^{1}$ model, while the gravitational descendants of the unity operator remain to be turned off, except for the $\sigma_{1}(\mathbf{1})$, which forms the condensate with $\left\langle\sigma_{1}(\mathbf{1})\right\rangle \neq 0$. An essential point is that string coupling $\hbar$ in the $\mathbb{P}^{1}$ model arises as certain "equivariant parameter" of the background, providing the infrared regularization of the theory on the gauge theory side [3], in order to collect contributions from the gauge theory instantons, while the instantonic expansion in gauge theory is going in powers of the scale $\Lambda^{2}=e^{\mathbf{t}^{\prime \prime}(a)}$.

The exact quasiclassical solution of this theory was explicitly constructed in [2] as a solution to dispersionless Toda hierarchy. More generally it was also proposed for the nonabelian extended Seiberg-Witten theory in terms of quasiclassical tau-function [4] on the deformed by ultraviolet perturbations Seiberg-Witten curve.

However, from the string side of duality this gives rise only to the truncated version of the $\mathbb{P}^{1}$ model, and a natural step would be including the whole set of descendants $\oplus_{k>0} T_{k} \sigma_{k}(\mathbf{1})$ of another primary - the unity operator. This has been done already in the $\mathbb{P}^{1}$ model itself, see $[5,6,7,8,9]$, where the matrix integral descriptions was first conjectured, the Virasoro constraints for the corresponding Gromov-Witten theory were formulated, and the generating functions were constructed in terms of specific correlators in the theory of free fermions.

Below we are going to write explicitly the quasiclassical solution to this theory, directly generalizing that of [2] (see also [12]). It terms of integrable hierarchies, it will raise the dispersionless Toda chain to the so called, following [10, 11], extended Toda hierarchy, where the gravitational descendants $\oplus_{k>0} T_{k} \sigma_{k}(\mathbf{1})$ of unity, and corresponding "logarithmic flows" [5] extend the set of mutually commuting flows of the Toda chain. It turns out, that introducing descendants of unity into the gauge theory is a very nontrivial step, presumably related to their role of "deformation" of the moduli space of background condensates in field theory, and we will find some hints of that reflected in the properties of the exact quasiclassical solution.

The extended quasiclassical solution will be constructed in pure geometric terms, which immediately suggest a natural nonabelian generalization - an extremely important thing if one would seriously have in mind the application of this duality for the purposes of gauge theory. The nonabelian generalization is also proposed below, but - quite typically in the geometric approach - only for class of solutions, when certain finite number of gravitational descendants of unity is turned on. We discuss also the relation of our solution to the variational problem for a 
certain functional (in spirit of $[2,13]$ ), in fact even with two equivalent functional formulations, whose exact relation with the Nekrasov partition function of summation over the gauge theory instantons [3] remains beyond the scope of this paper.

The paper is organized as follows: in sect. 2 we remind the construction for the quasiclassical solution to dispersionless Toda chain, corresponding to the half-truncated $\mathbb{P}^{1}$ topological string model, with the descendants of unity switched off, except for a condensate $\left\langle\sigma_{1}(\mathbf{1})\right\rangle \neq 0$. In sect. 3 we generalize this solution for the switched on descendants of unity, propose the formula for the first derivatives of the generating function w.r.t. new variables, and present explicit computations for the simplest nontrivial cases of this extension. Next, in sect. 4 we turn first time to the nonabelian theory, and construct the solution corresponding to the perturbative limit, which produces all important ingredients for the functional formulation of the problem: the kernel and generalized ultraviolet prepotential (1.1) for switched on descendants of unity. In sect. 5 we discuss the quasiclassical Virasoro constrains and functional formulations of the problem. Despite the form, suggested by perturbative nonabelian theory, we propose its equivalent formulation, obtained by an integral transformation and useful for studying the

dependence of the functional upon new times $\left\{T_{n}\right\}$ of the extended hierarchy. Finally, in sect. 6 we propose the formulation of the nonabelian $U\left(N_{c}\right)$ theory in terms of abelian differentials on hyperelliptic curve of genus $g=N_{c}-1$, and discuss the results and their possible generalizations in sect. 7.

\section{Dispersionless Toda chain}

Let us, first, remind the main formulas for the solution from [2] for the dispersionless Toda chain. We will follow here more convenient normalization from [12].

In the case of the deformed in the ultraviolet $U(1)$ supersymmetric gauge theory the $N_{c}=1$ Seiberg-Witten curve has a single cut, and the double cover of the $z$-plane $y^{2}=\left(z-x^{+}\right)\left(z-x^{-}\right)$ can be always presented in the form

$$
z=v+\Lambda\left(w+\frac{1}{w}\right)
$$

with $x^{ \pm}=v \pm 2 \Lambda$ and

$$
y^{2}=(z-v)^{2}-4 \Lambda^{2}
$$

The solution to dispersionless Toda chain is encoded into the function $S$, odd under the involution $w \leftrightarrow \frac{1}{w}$ on the double cover (2.1), with the asymptotic

$$
S(z) \underset{z \rightarrow \infty}{=}-2 z(\log z-1)+\mathbf{t}^{\prime}(z)+2 a \log z-\frac{\partial \mathcal{F}}{\partial a}-2 \sum_{k>0} \frac{1}{k z^{k}} \frac{\partial \mathcal{F}}{\partial t_{k}}
$$

The coefficients at singular terms are identified with the variables of the hierarchy, while the regular part of expansion defines the first derivatives of the (logarithm of the) tau-function $\mathcal{F}$. 
In terms of the uniformizing variable $w$ one can globally write

$$
S=-2 z \log w-2 \Lambda(\log \Lambda-1)\left(w-\frac{1}{w}\right)+\sum_{k>0} t_{k} \Omega_{k}(w)+2 a \log w
$$

where

$$
\Omega_{k}(w)=z_{+}^{k}-z_{-}^{k}, \quad k>0
$$

are the Laurent polynomials, odd under $w \leftrightarrow \frac{1}{w}$. The first term in (2.4) comes from the Legendre transform of the Seiberg-Witten differential $d \Sigma \sim z \frac{d w}{w}$.

The canonical Toda chain times are extracted from (2.3) by

$$
t_{0}=\operatorname{res}_{P_{+}} d S=-\operatorname{res}_{P_{-}} d S=2 a
$$

and

$$
t_{k}=\frac{1}{k} \operatorname{res}_{P_{+}} z^{-k} d S=-\frac{1}{k} \operatorname{res}_{P_{-}} z^{-k} d S, \quad k>0
$$

From the expansion of $S$ it also immediately follows, that

$$
\frac{\partial \mathcal{F}}{\partial t_{k}}=\frac{1}{2} \operatorname{res}_{P_{+}} z^{k} d S=-\frac{1}{2} \operatorname{res}_{P_{-}} z^{k} d S, \quad k>0
$$

The consistency condition for (2.8) is ensured by the symmetricity of second derivatives

$$
\frac{\partial^{2} \mathcal{F}}{\partial t_{n} \partial t_{k}}=\frac{1}{2} \operatorname{res}_{P_{+}}\left(z^{k} d \Omega_{n}\right)=\frac{1}{2} \operatorname{res}_{P_{+}}\left(z^{n} d \Omega_{k}\right)
$$

where

$$
\begin{gathered}
\Omega_{0}=\frac{\partial S}{\partial a} \underset{z \rightarrow P_{ \pm}}{=} \pm\left(2 \log z-\frac{\partial^{2} \mathcal{F}}{\partial a^{2}}-2 \sum_{n>0} \frac{\partial^{2} \mathcal{F}}{\partial a \partial t_{n}} \frac{1}{n z^{n}}\right) \\
\Omega_{k}=\frac{\partial S}{\partial t_{k}} \underset{z \rightarrow P_{ \pm}}{=} \pm\left(z^{k}-\frac{\partial^{2} \mathcal{F}}{\partial a \partial t_{k}}-2 \sum_{n>0} \frac{\partial^{2} \mathcal{F}}{\partial t_{k} \partial t_{n}} \frac{1}{n z^{n}}\right), \quad k>0
\end{gathered}
$$

form a basis of meromorphic functions with poles at the points $P_{ \pm}$, with $z\left(P_{ \pm}\right)=\infty$. All time-derivatives here are taken at constant $z$.

Expansion (2.10) of the Hamiltonian functions (2.5) expresses the second derivatives of $\mathcal{F}$ in terms of the coefficients of the equation of the curve (2.1), e.g.

$$
\begin{gathered}
\Omega_{0} \underset{z \rightarrow \infty}{=} 2 \log z-2 \log \Lambda-\frac{2 v}{z}-\frac{2 \Lambda^{2}+v^{2}}{z^{2}}+\ldots \\
\Omega_{1} \underset{z \rightarrow \infty}{=} z-v-\frac{2 \Lambda^{2}}{z}-\frac{2 v \Lambda^{2}}{z^{2}}+\ldots \\
\Omega_{2} \underset{z \rightarrow \infty}{=} z^{2}-\left(v^{2}+2 \Lambda^{2}\right)-\frac{4 v \Lambda^{2}}{z}-\frac{2 \Lambda^{2}\left(\Lambda^{2}+2 v^{2}\right)}{z^{2}}+\ldots
\end{gathered}
$$


Comparison of the coefficients in (2.11) gives, in particular,

$$
\frac{\partial^{2} \mathcal{F}}{\partial a^{2}}=\log \Lambda^{2}, \quad \frac{\partial^{2} \mathcal{F}}{\partial a \partial t_{1}}=v \quad \frac{\partial^{2} \mathcal{F}}{\partial t_{1}^{2}}=\Lambda^{2}
$$

and, therefore

$$
\frac{\partial^{2} \mathcal{F}}{\partial t_{1}^{2}}=\exp \frac{\partial^{2} \mathcal{F}}{\partial a^{2}}
$$

which becomes the long-wave limit of the Toda chain equations for the co-ordinate $a^{D}=\frac{\partial \mathcal{F}}{\partial a}$ after an extra derivative with respect to $a$ is taken

$$
\frac{\partial^{2} a^{D}}{\partial t_{1}^{2}}=\frac{\partial}{\partial a} \exp \frac{\partial a^{D}}{\partial a}
$$

One can now find the dependence of the coefficients of the curve (2.1) on the deformation parameters $\mathbf{t}$ of the microscopic theory by requiring $d S=0$ at the ramification points $z=$ $x_{ \pm}=v \pm 2 \Lambda$, where $d z=0$. This condition avoids from arising of extra singularities at the branch points in the variation of $d S$ w.r.t. moduli of the curve. Equation

$$
\frac{d z}{d \log w}=\Lambda\left(w-\frac{1}{w}\right)=0
$$

fixes the branch points to be at $w= \pm 1$, where now

$$
\left.\frac{d S}{d \log w}\right|_{w= \pm 1}=\left.\sum_{k>0} t_{k} \frac{d \Omega_{k}}{d \log w}\right|_{w= \pm 1}+2 a-2 v \mp 4 \Lambda \log \Lambda=0
$$

If $t_{k}=0$ for $k>1$, solution to (2.16) immediately gives

$$
v=a, \quad \Lambda^{2}=e^{t_{1}}
$$

and the prepotential

$$
\mathcal{F}=\frac{1}{2} a a^{D}+\frac{1}{2} \operatorname{res}_{P_{+}}(z d S)-\frac{a^{2}}{2}=\frac{1}{2} a^{2} t_{1}+e^{t_{1}}
$$

which is a well-known expression for the generating function of the $\mathbb{P}^{1}$ model, restricted to the "small phase space" of the primary operators.

\section{$\Phi$-function}

In the context of dispersionless and generic quasiclassical hierarchies it is useful to introduce

$$
\Phi=\frac{d S}{d z} \underset{z \rightarrow \infty}{=}-2 \log z+\mathbf{t}^{\prime \prime}(z)+\frac{2 a}{z}+2 \sum_{k>0} \frac{1}{z^{k+1}} \frac{\partial \mathcal{F}}{\partial t_{k}}
$$


odd under the involution $w \leftrightarrow \frac{1}{w}$, or globally

$$
\Phi=-2 \log w+\sum_{k>1} k t_{k} \Omega_{k-1}
$$

Consistency between (2.20) and (2.19) gives rise exactly to the equations (2.16), and can be used as another way of their derivation. This function does not have singularities except for the points $P_{ \pm}$with $z\left(P_{ \pm}\right)=\infty$. It has a natural integral representation

$$
\Phi(z)=\mathbf{t}^{\prime \prime}(z)-\int d x f^{\prime \prime}(x) \log (z-x)
$$

with the integrable "density" $f^{\prime \prime}(x)$

$$
\frac{1}{2} \int d x f^{\prime \prime}(x)=1, \quad \frac{1}{2} \int d x x f^{\prime \prime}(x)=a
$$

related to the second derivative of the extremal shape function for random partitions [13]. One can easily see, that

$$
2 i f^{\prime \prime}(z)=\Delta \Phi^{\prime}(z)=\Phi^{\prime}(z+i 0)-\Phi^{\prime}(z-i 0)
$$

while for the function (2.21) itself one gets

$$
\Delta \Phi(z)=\Phi(z+i 0)-\Phi(z-i 0)=-2 i \int d x f^{\prime \prime}(x) \arg (z-x)=2 i f^{\prime}(z)
$$

The function $\Phi$, together with $z$ (or generally one should better refer to their differentials $d \Phi$ and $d z[4]$ ), is a basic ingredient for the quasiclassical hierarchy, and will be exploited below, when discussing the Virasoro constrants.

\section{$3 \quad$ Extended quasiclassical Toda hierarchy}

Formula (2.4) can be naturally generalized to the higher logarithmic flows

$$
S=\sum_{k>0} t_{k} \Omega_{k}(w)+2 a \log w-2 \sum_{n>0} T_{n} H_{n}(z, w)
$$

so that $(2.4)$ is a particular case of $(3.1)$, corresponding to $T_{n}=\delta_{n, 1}$. The extra Hamiltonians

$$
H_{k}(z, w)=z^{k} \log w+\sum_{j=1}^{k} C_{j}^{(k)} \Omega_{j}(w)
$$


are odd under involution $w \leftrightarrow \frac{1}{w}$ and fixed by the asymptotic

$$
\begin{gathered}
H_{k}(z, w) \underset{z \rightarrow \infty}{=} \pm H_{k}^{(+)}(z)+O(1) \\
H_{k}^{(+)}(z)=z^{k}\left(\log z-c_{k}\right)
\end{gathered}
$$

where the Harmonic numbers

$$
c_{k}=\sum_{i=1}^{k} \frac{1}{i}, \quad k>0
$$

(one can also set $c_{0}=0$ ) ensure "scaling property" of the singular parts

$$
d H_{k}^{(+)}=k H_{k-1}^{(+)} d z
$$

From (3.3) one immediately gets, that

$$
\begin{gathered}
C_{k}^{(k)}=\log \Lambda-c_{k}=H_{k}^{(+)}(\Lambda) \Lambda^{-k} \\
C_{j}^{(k)}=\omega_{k-j}, \quad j=1, \ldots, k-1 \\
\log w \underset{z=\infty}{=} \log z-\log \Lambda-\sum_{k>0} \frac{\omega_{k}}{z^{k}}
\end{gathered}
$$

In particular, $H_{0}=\log w$, and

$$
H_{1}(z, w)=z \log w+\Lambda(\log \Lambda-1)\left(w-\frac{1}{w}\right)
$$

is the Eguchi-Yang term (see [5]), remaining in the expansion $(2.3)$ for $T_{n}=\delta_{n, 1}$, which corresponds to nonvanishing condensate $\left\langle\sigma_{1}(\mathbf{1})\right\rangle \neq 0$. One can also write for $(3.1)$

$$
S=-2 T(z) \log w+2 a \log w+\sum_{k>0} \hat{t}_{k} \Omega_{k}(w)
$$

with

$$
\begin{gathered}
T(z)=\sum_{n>0} T_{n} z^{n} \\
\hat{t}_{k}=t_{k}-2 T_{k}\left(\log \Lambda-c_{k}\right)-2 \sum_{l>0} \omega_{l} T_{k+l}, \quad k>0
\end{gathered}
$$

which can be interpreted as a reparameterization $z \rightarrow T(z)$ with certain compensating transformation of the function (2.4). The function $T(z)$, and therefore the times $\left\{T_{n}\right\}$ can be defined through the jumps of the function (3.1), (3.8)

$$
T(z)=\frac{i}{4 \pi} \Delta S
$$


or, in a different way, via the residues of derivatives

$$
T_{n}=-\frac{1}{2 n !} \operatorname{res}_{P_{+}} d S^{(n)}=\frac{1}{2 n !} \operatorname{res}_{P_{-}} d S^{(n)}, \quad n \geq 0
$$

with $S^{(n)}=\frac{d^{n} S}{d z^{n}}$.

Now let us propose the dual to (3.11) formula, which defines the corresponding derivatives of the prepotential

$$
\left.\frac{\partial \mathcal{F}}{\partial T_{n}}\right|_{\mathbf{t}}=(-)^{n} n !\left(S_{n}\right)_{0}
$$

where

$$
\frac{d^{n} S_{n}}{d z^{n}}=S, \quad n \geq 0
$$

or $S_{n}$ is the $n$-th primitive of (3.1), odd under the involution $w \leftrightarrow \frac{1}{w}$ of $(2.1)$. This is a new ingredient in the formulation of quasiclassical hierarchy, going beyond the original setup of [4]. This formula is directly related to the gravitational dressing of the primary operators in the (here dual, with the superpotential $z=v+\Lambda\left(w+\frac{1}{w}\right)$ on the $w$-cylinder) LandauGinzburg theory, suggested in [14]. We propose now, that (3.12), (3.13) is a strict definition of dependence of the quasiclassical tau-function upon the times if extended hierarchy, which is trusted by symmetricity of the corresponding second derivatives of (3.14) and (3.12), following from the Riemann bilinear identities on the cut $w$-cylinder (2.1), see Appendix. The definitions of the prepotential, as a function of Toda chain times $\mathbf{t}$ remains intact, i.e.

$$
\left.\frac{\partial \mathcal{F}}{\partial t_{k}}\right|_{\mathbf{T}}=\frac{1}{2} \operatorname{res}_{P_{+}} z^{k} d S=-\frac{1}{2} \operatorname{res}_{P_{-}} z^{k} d S, \quad k>0
$$

where the derivatives are now taken at fixed $\mathbf{T}$.

Instead of (2.3) one can now write for (3.1)

$$
S(z) \underset{z \rightarrow \infty}{=}-2 \sum_{n>0} T_{n} z^{n}\left(\log z-c_{n}\right)+\mathbf{t}^{\prime}(z)+2 a \log z-\frac{\partial \mathcal{F}}{\partial a}-2 \sum_{k>0} \frac{1}{k z^{k}} \frac{\partial \mathcal{F}}{\partial t_{k}}
$$

It means, that in addition to (2.10) one gets for the logarithmic Hamiltonians

$$
H_{n}(z, w)=-\frac{1}{2} \frac{\partial S(z)}{\partial T_{n}} \underset{z \rightarrow \infty}{=} z^{n}\left(\log z-c_{n}\right)+\frac{1}{2} \frac{\partial^{2} \mathcal{F}}{\partial a \partial T_{n}}+\sum_{k>0} \frac{1}{k z^{k}} \frac{\partial^{2} \mathcal{F}}{\partial T_{n} \partial t_{k}}
$$

Note also, that the constant term in the r.h.s. of (3.12) essentially depend on the negative powers of expansions of $\Omega_{k}$, therefore $\frac{\partial \mathcal{F}}{\partial T_{n}}$ is expressed in terms of $\frac{\partial^{2} \mathcal{F}}{\partial t_{k} \partial t_{n}}$, and this can be rewritten as a sort of quasiclassical mixed Hirota-Virasoro type constraints. For example, one gets in this way

$$
d S_{1}=S(z) d z=\sum_{k>0} t_{k} z^{k} d z+2 a \log z d z-2 \sum_{n>o} T_{n} H_{n}^{(+)}(z) d z-\frac{\partial \mathcal{F}}{\partial a} d z-2 \frac{\partial \mathcal{F}}{\partial t_{1}} \frac{d z}{z}+\ldots
$$


i.e.

$$
S_{1}=\sum_{k>0} \frac{t_{k}}{k+1} \Omega_{k+1}(w)+2 a H_{1}(z, w)-2 \sum_{n>0} \frac{T_{n}}{n+1} H_{n+1}(z, w)-\frac{\partial \mathcal{F}}{\partial a} \Omega_{1}(w)-2 \frac{\partial \mathcal{F}}{\partial t_{1}} \log w
$$

and therefore

$$
\left(S_{1}\right)_{0}=-\sum_{k>0} \frac{t_{k}}{k+1} \frac{\partial^{2} \mathcal{F}}{\partial a \partial t_{k+1}}+a \frac{\partial^{2} \mathcal{F}}{\partial a \partial T_{1}}-\sum_{n>o} \frac{T_{n}}{n+1} \frac{\partial^{2} \mathcal{F}}{\partial a \partial T_{n+1}}+\frac{\partial \mathcal{F}}{\partial a} \frac{\partial^{2} \mathcal{F}}{\partial a \partial t_{1}}+\frac{\partial \mathcal{F}}{\partial t_{1}} \frac{\partial^{2} \mathcal{F}}{\partial a^{2}}
$$

Upon (3.12) this can be rewritten as

$$
\frac{\partial}{\partial a}\left(a \frac{\partial \mathcal{F}}{\partial T_{1}}+\frac{\partial \mathcal{F}}{\partial a} \frac{\partial \mathcal{F}}{\partial t_{1}}-\sum_{k>0} \frac{1}{k+1}\left(t_{k} \frac{\partial \mathcal{F}}{\partial t_{k+1}}+T_{k} \frac{\partial \mathcal{F}}{\partial T_{k+1}}\right)\right)=0
$$

The quasiclassical Virasoro constraints in their canonical form will be discussed below in sect. 5 .

Small phase space

Let now only $t_{1}, a$ and $T_{1} \neq 1$ be nonvanishing. Then

$$
\begin{gathered}
S=t_{1} \Omega_{1}+2 a \log w-2 T_{1} H_{1}= \\
\underset{z \rightarrow \infty}{=} t_{1} z-2 T_{1} z(\log z-1)+2 a \log z+\left(2 T_{1} v \log \Lambda-t_{1} v-2 a \log \Lambda\right)- \\
-\left(2 T_{1} \Lambda^{2}-T_{1} v^{2}-4 T_{1} \Lambda^{2} \log \Lambda+2 T_{1} \Lambda^{2}+2 a v\right) \frac{1}{z}+O\left(\frac{1}{z^{2}}\right)
\end{gathered}
$$

which means that

$$
\begin{aligned}
S_{1}=\frac{t_{1}}{2} \Omega_{2}(w) & -T_{1} H_{2}(z, w)+2 a H_{1}(z, w)+\left(2 T_{1} v \log \Lambda-t_{1} v-2 a \log \Lambda\right) \Omega_{1}(w)- \\
& -\left(2 T_{1} \Lambda^{2}-T_{1} v^{2}-4 T_{1} \Lambda^{2} \log \Lambda+2 T_{1} \Lambda^{2}+2 a v\right) \log w
\end{aligned}
$$

and therefore

$$
\begin{gathered}
\left(S_{1}\right)_{0}=\frac{1}{2} t_{1} v^{2}-t_{1} \Lambda^{2}-2 T_{1} \Lambda^{2}-2 T_{1} v^{2} \log \Lambda+4 T_{1} \Lambda^{2} \log \Lambda+2 a v \log \Lambda- \\
-4 T_{1} \Lambda^{2}(\log \Lambda)^{2}+2 t_{1} \Lambda^{2} \log \Lambda
\end{gathered}
$$

Equations $\left.\frac{d S}{d \log w}\right|_{w= \pm 1}=0$ now give

$$
v=\frac{a}{T_{1}}, \quad \Lambda^{2}=\exp \frac{t_{1}}{T_{1}}
$$


which upon substitution into (3.23), and using (3.12) gives rise to

$$
\mathcal{F}\left(t_{1}, a, T_{1}\right)=\frac{a^{2} t_{1}}{2 T_{1}}+T_{1}^{2} \exp \frac{t_{1}}{T_{1}}
$$

found originally in [6]. One can conclude therefore, that switching on the first time $T_{1}$ results in simultaneous rescaling of all the times $t_{1} \rightarrow \frac{t_{1}}{T_{1}}, a \rightarrow \frac{a}{t_{1}}$ etc, together with the string coupling $\hbar \rightarrow \frac{\hbar}{T_{1}}$, since $(3.25)$ can be rewritten as

$$
\frac{1}{T_{1}^{2}} \mathcal{F}\left(t_{1}, a, T_{1}\right)=\frac{1}{2}\left(\frac{a}{T_{1}}\right)^{2} \frac{t_{1}}{T_{1}}+\exp \frac{t_{1}}{T_{1}}=\mathcal{F}\left(\frac{t_{1}}{T_{1}}, \frac{a}{T_{1}} ; T_{1}=1\right)
$$

with the r.h.s. defined in (2.18).

It is interesting to point out that at $T_{1} \rightarrow \infty,(3.25)$ gives

$$
\begin{gathered}
\mathcal{F}\left(t_{1}, a, T_{1}\right) \underset{T_{1} \rightarrow \infty}{\sim}\left(T_{1}^{2}+T_{1} t_{1}+\frac{t_{1}^{2}}{2}\right)+\frac{1}{T_{1}}\left(\frac{a^{2} t_{1}}{2}+\frac{t_{1}^{3}}{6}\right)+\ldots= \\
=\ldots+\frac{1}{6 T_{1}}\left(\left(t_{1}+a\right)^{3}+\left(t_{1}-a\right)^{3}\right)+\ldots=\ldots \mathrm{F}\left(t_{1}+a, T_{1}\right)+\mathrm{F}\left(t_{1}-a, T_{1}\right)+\ldots
\end{gathered}
$$

modulo quadratic terms and $O\left(T_{1}^{-2}\right)$, where

$$
\mathrm{F}\left(x, T_{1}\right)=\frac{x^{3}}{6 T_{1}}
$$

is the prepotential of pure two-dimensional topological gravity.

$T_{2}$ now switched on

Equations (2.16) for the switched on $T_{2}$ (in addition to the small phase space) give rise to

$$
\begin{gathered}
t_{1}=\left(2 T_{1}+4 T_{2} v\right) \log \Lambda \\
a=T_{1} v+T_{2}\left(v^{2}-2 \Lambda^{2}+4 \Lambda^{2} \log \Lambda\right)
\end{gathered}
$$

which already cannot be solved analytically for $v$ and $\Lambda$, though the solutions can be easily found as series in $T_{2}$, with the first few terms

$$
\begin{gathered}
v=\frac{a}{T_{1}}-\frac{T_{2}}{T_{1}^{3}}\left(a^{2}+2 t_{1} T_{1} e^{\frac{t_{1}}{T_{1}}}-2 T_{1}^{2} e^{\frac{t_{1}}{T_{1}}}\right)+\frac{2 a T_{2}^{2}}{T_{1}^{5}}\left(a^{2}+2 t_{1}^{2} e^{\frac{t_{1}}{T_{1}}}+2 t_{1} T_{1} e^{\frac{t_{1}}{T_{1}}}-2 T_{1}^{2} e^{\frac{t_{1}}{T_{1}}}\right)+\ldots \\
\log \Lambda=\frac{t_{1}}{2 T_{1}}-\frac{a t_{1} T_{2}}{T_{1}^{3}}+\frac{t_{1} T_{2}^{2}}{T_{1}^{5}}\left(3 a^{2}+2 t_{1} T_{1} e^{\frac{t_{1}}{T_{1}}}-2 T_{1}^{2} e^{\frac{t_{1}}{T_{1}}}\right)+\ldots
\end{gathered}
$$


and formulas (3.14), (3.12) lead to the following expression for the prepotential

$$
\begin{gathered}
\mathcal{F}=\frac{a^{2} t_{1}}{2 T_{1}}+T_{1}^{2} e^{\frac{t_{1}}{T_{1}}}+T_{2}\left(-\frac{a^{3} t_{1}}{3 T_{1}^{3}}+4 a e^{\frac{t_{1}}{T_{1}}}-\frac{2 a t_{1}}{T_{1}} e^{\frac{t_{1}}{T_{1}}}\right)+ \\
+T_{2}^{2}\left(\frac{a^{4} t_{1}}{2 T_{1}^{5}}+\frac{2 a^{2} t_{1}^{2}}{T_{1}^{4}} e^{\frac{t_{1}}{T_{1}}}-\frac{2 a^{2} t_{1}}{T_{1}^{3}} e^{\frac{t_{1}}{T_{1}}}+\frac{t_{1}^{2}}{T_{1}^{2}} e^{\frac{2 t_{1}}{T_{1}}}-\frac{3 t_{1}}{T_{1}} e^{\frac{2 t_{1}}{T_{1}}}+\frac{5}{2} e^{\frac{2 t_{1}}{T_{1}}}\right)+\ldots
\end{gathered}
$$

which certainly satisfies, up to quadratic order in $T_{2}$, the long-wave limit of the Toda chain equation (2.13). One can also easily check, that formula (3.31) up to the shift $T_{1} \rightarrow 1-\delta T_{1}$ from the condensate of $\left\langle\sigma_{1}(\mathbf{1})\right\rangle$ and certain rescaling (say, $T_{2} \rightarrow-\frac{T_{2}}{2}$ ) coincides with the expansion, obtained in the Appendix of the second paper of [10]. When deriving (3.31) we have used, in particular, $n=1,2$ cases of (3.12), expressing as in (3.23) the constant parts of the first two primitives of $S$ (together with the constant part of the $S$ itself) in terms of the coefficients of the curve $(2.1)$

$$
\begin{gathered}
(S)_{0}=2 T_{1} v \log \Lambda-4 T_{2} \Lambda^{2}+2 T_{2} v^{2} \log \Lambda+4 T_{2} \Lambda^{2} \log \Lambda-v t_{1}-2 a \log \Lambda \\
\left(S_{1}\right)_{0}=4 T_{2} v \Lambda^{2} \log \Lambda-4 T_{1} \Lambda^{2}(\log \Lambda)^{2}+4 T_{1} \Lambda^{2} \log \Lambda-2 T_{2} v^{3} \log \Lambda-2 T_{1} v^{2} \log \Lambda+ \\
+\frac{1}{2} t_{1} v^{2}-2 \Lambda^{2} T_{1}+2 a v \log \Lambda-8 v \Lambda^{2} T_{2}(\log \Lambda)^{2}+2 t_{1} \Lambda^{2} \log \Lambda-t_{1} \Lambda^{2} \\
\left(S_{2}\right)_{0}=T_{2} v^{4} \log \Lambda-2 a \Lambda^{2} \log \Lambda-a v^{2} \log \Lambda+2 a \Lambda^{2}+t_{1} \Lambda^{2} v-\frac{1}{6} t_{1} v^{3}+ \\
+T_{1} v^{3} \log \Lambda+\frac{5}{2} T_{2} \Lambda^{4}-2 T_{1} v \Lambda^{2} \log \Lambda-4 T_{2} v^{2} \Lambda^{2} \log \Lambda-6 T_{2} \Lambda^{4} \log \Lambda+ \\
+4 T_{2} \Lambda^{4}(\log \Lambda)^{2}+8 T_{2} v^{2} \Lambda^{2}(\log \Lambda)^{2}+4 T_{1} v \Lambda^{2}(\log \Lambda)^{2}-2 t_{1} v \Lambda^{2} \log \Lambda
\end{gathered}
$$

It is also instructive to write explicitly in this case

$$
\begin{gathered}
\Phi\left(t_{1}, a, T_{1}, T_{2}\right)=\frac{d S}{d z}=-2 T_{1} \log w-4 T_{2} H_{1}(z, w)= \\
=-2 T_{1} \log w-4 T_{2}\left(z \log w+\Lambda(\log \Lambda-1)\left(w-\frac{1}{w}\right)\right)
\end{gathered}
$$

and

$$
\Phi^{\prime}\left(t_{1}, a, T_{1}, T_{2}\right)=\frac{d \Phi}{d z}=-4 T_{2} \log w-\frac{2}{\Lambda} \frac{T_{1}+2 T_{2} v+2 T_{2} \log \Lambda(z-v)}{w-\frac{1}{w}}
$$

where the coefficients of the curve (2.1) $\Lambda=\Lambda\left(t_{1}, a, T_{1}, T_{2}\right)$ and $v=v\left(t_{1}, a, T_{1}, T_{2}\right)$ are constrained by (3.29). We see, in particular, that the Vershik-Kerov "arcsin law" [15], corresponding to the first term in the r.h.s. of (3.33) is now not only perturbed by the semicircle Wigner distribution, (like for the $\sigma_{1}(\varpi)$ or $t_{2}$ switched on, see $[2,12]$ ), but is also "modulated" by multiplication by a linear function. Moreover, one can find, that

$$
\begin{gathered}
\Phi^{\prime \prime}\left(t_{1}, a, T_{1}, T_{2}\right)=\frac{d^{2} \Phi}{d z^{2}}= \\
=\frac{1}{\sqrt{(z-v)^{2}-4 \Lambda^{2}}}\left(-4 T_{2}+\frac{T_{1}+2 T_{2} v+4 T_{2} \Lambda \log \Lambda}{z-v-2 \Lambda}+\frac{T_{1}+2 T_{2} v-4 T_{2} \Lambda \log \Lambda}{z-v+2 \Lambda}\right)
\end{gathered}
$$


For the nonabelian generalization it is also rather useful to rewrite (3.34) in the form

$$
d \Phi\left(t_{1}, a, T_{1}, T_{2}\right)=-4 T_{2} \log w d z-4 T_{2} \log \Lambda d y-2\left(T_{1}+2 T_{2} v\right) \frac{d z}{y}
$$

with $y$ defined in $(2.2)$.

$T_{2}, T_{3}$ switched on

Now, instead of (3.33), one gets

$$
\Phi\left(t_{1}, a, T_{1}, T_{2}, T_{3}\right)=\frac{d S}{d z}=-2 T_{1} \log w-4 T_{2} H_{1}(z, w)-6 T_{3} H_{2}(z, w)
$$

provided by

$$
\begin{gathered}
t_{1}=\left(2 T_{1}+4 T_{2} v+6 T_{3} v^{2}\right) \log \Lambda+12 T_{3} \Lambda^{2}(\log \Lambda-1) \\
a=T_{1} v+T_{2}\left(v^{2}-2 \Lambda^{2}+4 \Lambda^{2} \log \Lambda\right)+T_{3}\left(v^{3}-6 v \Lambda^{2}+12 v \Lambda^{2} \log \Lambda\right)
\end{gathered}
$$

One can easily notice, that in the limit suppressing instantons, i.e. suppressing powers of $\Lambda$ and keeping only the $\log$ arithmic terms $\log \Lambda$, equations (3.29), (3.38) acquire the form

$$
\begin{gathered}
t_{1}=2 T^{\prime}(v) \log \Lambda+O\left(\Lambda^{2}\right) \\
a=T(v)+O\left(\Lambda^{2}\right)
\end{gathered}
$$

reflecting the sense of higher descendants of unity as reparameterization $z \rightarrow T(z)$.

\section{Nonabelian theory: perturbative limit}

Let us now turn to the problem, how to construct the abelian integral with asymptotic (3.15) on generic hyperelliptic curve

$$
y^{2}=\prod_{j=1}^{2 N_{c}}\left(z-x_{j}\right)
$$

of the extended nonabelian Seiberg-Witten theory. On the small phase space, i.e. when only the $t_{1}$ is nonvanishing, or the descendants of the Kähler class are switched off, the curve (4.1) can be also written as

$$
\Lambda^{N_{c}}\left(w+\frac{1}{w}\right)=P_{N_{c}}(z)=\prod_{i=1}^{N_{c}}\left(z-v_{i}\right)
$$

with (4.1) turning into

$$
y^{2}=P_{N_{c}}(z)^{2}-4 \Lambda^{2 N_{c}}
$$


The perturbative limit corresponds to $\Lambda \rightarrow 0$ in the above formulas, when the hyperelliptic curve splits into two disjoint sheets of $z$-plane with $N_{c}$ punctures, which can be described by

$$
w_{\text {pert }}=P_{N_{c}}(z)=\prod_{i=1}^{N_{c}}\left(z-v_{i}\right)
$$

i.e. a rational function on the $z$-plane with $N_{c}$ punctures. In this section we discuss the perturbative limit of the nonabelian theory, defined entirely in terms of the rational curve (4.4), and turn to generic situation of (4.1) below in sect. 6.

Only $T_{2}$ switched on

A perturbative anzatz for

$$
\Phi^{\prime}=-2 \sum_{j=1}^{N_{c}}\left(2 T_{2} \log \left(z-v_{j}\right)+\frac{T^{\prime}\left(v_{j}\right)}{z-v_{j}}\right)
$$

with

$$
T^{\prime}\left(v_{j}\right)=T_{1}+2 T_{2} v_{j}, \quad j=1, \ldots, N_{c}
$$

can be easily conjectured, having e.g. formula (3.36). The coefficients of (4.5) are fixed by

$$
\begin{gathered}
\operatorname{res}_{z=\infty} d \Phi^{\prime}=4 T_{2} \cdot N_{c} \\
\operatorname{res}_{z=\infty} d \Phi \equiv-\operatorname{res}_{z=\infty} d \Phi^{\prime}=-2 T_{1} \cdot N_{c}
\end{gathered}
$$

The modified Seiberg-Witten periods are now given by the formulas

$$
\begin{aligned}
& a_{j}=\frac{1}{2 \pi i} \oint_{A_{j}} \frac{z^{2}}{2} d \Phi^{\prime}=\operatorname{res}_{z=v_{j}} \frac{z^{2}}{2} d \Phi^{\prime}= \\
& =T\left(v_{j}\right)=T_{1} v_{j}+T_{2} v_{j}^{2}, \quad j=1, \ldots, N_{c}
\end{aligned}
$$

Integrating (4.5) one gets explicitly

$$
\Phi=-2 \sum_{j=1}^{N_{c}}\left(2 T_{2}\left(z-v_{j}\right)\left(\log \left(z-v_{j}\right)-1\right)+T^{\prime}\left(v_{j}\right) \log \left(z-v_{j}\right)\right)+t_{1}
$$

One easily finds, that the derivatives of generating differential

$$
\frac{\partial}{\partial a_{j}} \Phi d z=\frac{1}{T^{\prime}\left(v_{j}\right)} \frac{\partial \Phi}{\partial v_{j}} d z=2 \frac{d z}{z-v_{j}}, \quad j=1, \ldots, N_{c}
$$


appear to be the "canonical holomorphic" differentials with the first order poles at $z=v_{j}$ on rational degeneration of the curve (4.2). Moreover, one can find, that

$$
-2 d H_{n}=\left.\frac{\partial}{\partial T_{n}} \Phi\right|_{a} d z=\left.\frac{\partial}{\partial T_{n}} \Phi\right|_{v} d z-\sum_{j=1}^{N_{c}} v_{j}^{n} \frac{d z}{z-v_{j}}
$$

giving rise to

$$
\begin{gathered}
d H_{1}=d z \sum_{j=1}^{N_{c}}\left(\log \left(z-v_{j}\right)+\frac{v_{j}}{z-v_{j}}\right) \\
d H_{2}=d z \sum_{j=1}^{N_{c}}\left(2 z\left(\log \left(z-v_{j}\right)-1\right)+2 v_{j}+\frac{v_{j}^{2}}{z-v_{j}}\right)
\end{gathered}
$$

where the last terms in the r.h.s. (linear combinations of the "holomorphic" differentials on degenerate rational curve) kill the residue at infinity.

Integrating (4.9) further, one finds

$$
\begin{gathered}
S=t_{1} z-2 \sum_{j=1}^{N_{c}}\left(T_{2}\left(z-v_{j}\right)^{2}\left(\log \left(z-v_{j}\right)-\frac{3}{2}\right)+T^{\prime}\left(v_{j}\right)\left(z-v_{j}\right)\left(\log \left(z-v_{j}\right)-1\right)\right)= \\
=t_{1} z-2 \sum_{j=1}^{N_{c}}\left(T_{2} H_{2}^{(+)}\left(z-v_{j}\right)+T^{\prime}\left(v_{j}\right) H_{1}^{(+)}\left(z-v_{j}\right)\right)
\end{gathered}
$$

which defines the perturbative prepotential by

$$
a_{i}^{D}=S\left(v_{i}\right)=t_{1} v_{i}-2 \sum_{j \neq i}\left(T_{2} H_{2}^{(+)}\left(v_{i}-v_{j}\right)+T^{\prime}\left(v_{j}\right) H_{1}^{(+)}\left(v_{i}-v_{j}\right)\right)=\frac{\partial \mathcal{F}_{\text {pert }}}{\partial a_{i}}
$$

Formula (4.14) can be integrated, since for $i \neq k$ one gets

$$
\frac{\partial S\left(v_{i}\right)}{\partial v_{k}}=-2 T^{\prime}\left(v_{k}\right) \log \left(v_{i}-v_{k}\right)
$$

and this gives rise to the perturbative prepotential

$$
\mathcal{F}_{\text {pert }}\left(a_{1}, \ldots, a_{N_{c}} ; T_{1}, T_{2}\right)=\sum_{j=1}^{N_{c}} F_{U V}\left(v_{j}\right)+\sum_{i \neq j} F\left(v_{i}, v_{j} ; T_{1}, T_{2}\right)
$$

where one have substitute for $v_{i}$ a solution to $T\left(v_{i}\right)=a_{i}$ with the asymptotic $v_{i} \sim \frac{a_{i}}{T_{1}}+\ldots$, when expanding over the higher times $T_{n}$. The bare ultraviolet prepotential

$$
F_{U V}(v)=\frac{1}{2} t_{1}\left(T_{1} v^{2}+\frac{4}{3} T_{2} v^{2}\right)=\frac{a^{2} t_{1}}{2 T_{1}}-\frac{T_{2} a^{3} t_{1}}{3 T_{1}^{3}}+\frac{T_{2}^{2} a^{4} t_{1}}{2 T_{1}^{5}}+\ldots
$$


coincides, of course, with the perturbative part of the $U(1)$ prepotential (3.31) or partition function of the $\mathbb{P}^{1}$ model. The "interacting part" in (4.19) $F\left(v_{i}, v_{j} ; T_{1}, T_{2}\right)$ satisfies the integrability condition

$$
\frac{\partial^{2} F}{\partial a_{i} \partial a_{j}}=\log \left(v_{i}-v_{j}\right)
$$

provided by $a_{i}=T\left(v_{i}\right), i=1, \ldots, N_{c}$. If only $T_{1}, T_{2} \neq 0$, the direct integration gives an expression

$$
\begin{gathered}
F\left(v_{1}, v_{2} ; T_{1}, T_{2}\right)=-\frac{1}{2}\left(v_{1}-v_{2}\right)^{2}\left(T_{1}+T_{2}\left(v_{1}+v_{2}\right)\right)^{2} \log \left(v_{1}-v_{2}\right)+ \\
+\frac{1}{4}\left(v_{1}-v_{2}\right)^{2}\left(3\left(T_{1}+T_{2}\left(v_{1}+v_{2}\right)\right)^{2}+\frac{1}{2} T_{2}^{2}\left(v_{1}-v_{2}\right)^{2}\right)= \\
=-\frac{1}{2}\left(T\left(v_{1}\right)-T\left(v_{2}\right)\right)^{2} \log \left(v_{1}-v_{2}\right)+\frac{3}{4}\left(T\left(v_{1}\right)-T\left(v_{2}\right)\right)^{2}+\frac{T_{2}^{2}}{8}\left(v_{1}-v_{2}\right)^{4}= \\
=-\frac{1}{2}\left(a_{1}-a_{2}\right)^{2} \log \left(v_{1}-v_{2}\right)+\frac{3}{4}\left(a_{1}-a_{2}\right)^{2}+\frac{T_{2}^{2}}{8}\left(v_{1}-v_{2}\right)^{4}
\end{gathered}
$$

Expanding over $T_{2}$ we see that gravitational descendants of unity give rise to the polynomial corrections to the coupling constants

$$
T_{i j}=\frac{\partial^{2} \mathcal{F}_{\text {pert }}}{\partial a_{i} \partial a_{j}} \sim \log \left(v_{i}-v_{j}\right)=\log \frac{a_{i}-a_{j}}{T_{1}}-\frac{T_{2}}{T_{1}^{2}}\left(a_{i}+a_{j}\right)+O\left(T_{2}^{2}\right)
$$

which remind arising in the context of five-dimensional supersymmetric gauge theories. Moreover, for the particular values $T_{n}=\frac{(-)^{n-1}}{n}$, we get formally the perturbative limit of the (compactified) five-dimensional Seiberg-Witten theory [16], with the infrared couplings

$$
\log \left(v_{i}-v_{j}\right)=\log \left(e^{a_{i}}-e^{a_{j}}\right)=\frac{a_{i}+a_{j}}{2}+\log \left(2 \sinh \frac{a_{i}-a_{j}}{2}\right)
$$

and studied recently in the context of its relation to summing over random partitions in [17].

Perturbative theory with $N$ descendants of unity switched on

For $N$ first descendants of unity switched on (with arbitrary $N$ ), it is convenient to introduce auxiliary functions

$$
\begin{gathered}
\sigma(z ; x)=\sum_{k>0} \frac{T^{(k)}(x)}{k !} H_{k}^{(+)}(z-x) \\
\varphi(z ; x)=\frac{d \sigma}{d z}=\sum_{k>1} \frac{T^{(k)}(x)}{(k-1) !} H_{k-1}^{(+)}(z-x)+T^{\prime}(x) \log (z-x)
\end{gathered}
$$


with $T^{(k)}(x)$ being $k$-th derivatives of the polynomial $T(x)=\sum_{n=1}^{N} T_{n} x^{n}$. One can define generally

$$
\begin{gathered}
S(z)=S\left(z ; v_{1}, \ldots, v_{N_{c}}\right)=-2 \sum_{j=1}^{N_{c}} \sigma\left(z ; v_{j}\right)+\mathbf{t}^{\prime}(z) \\
\Phi(z)=\Phi\left(z ; v_{1}, \ldots, v_{N_{c}}\right)=-2 \sum_{j=1}^{N_{c}} \varphi\left(z ; v_{j}\right)+\mathbf{t}^{\prime \prime}(z)=\frac{d S}{d z}
\end{gathered}
$$

and express the derivatives of the perturbative prepotential as

$$
\frac{\partial \mathcal{F}_{\text {pert }}}{\partial v_{i}}=S\left(v_{i}\right)=\mathbf{t}^{\prime}\left(v_{i}\right)-2 \sum_{j \neq i} \sigma\left(v_{i} ; v_{j}\right)
$$

where the integrability condition (4.18) is now ensured by

$$
\begin{gathered}
\frac{\partial}{\partial x} \sigma(z ; x)=\sum_{k>0} \frac{T^{(k+1)}(x)}{k !} H_{k}^{(+)}(z-x)-\sum_{k>0} \frac{T^{(k)}(x)}{(k-1) !} H_{k-1}^{(+)}(z-x)= \\
=\frac{T^{(N+1)}(x)}{N !} H_{N}^{(+)}(z-x)-T^{\prime}(x) \log (z-x)=-T^{\prime}(x) \log (z-x)
\end{gathered}
$$

We therefore justify formula (4.19) for arbitrary $N$, i.e.

$$
\begin{gathered}
\mathcal{F}_{\text {pert }}\left(a_{1}, \ldots, a_{N_{c}} ; \mathbf{t}, T\right)=\sum_{j=1}^{N_{c}} F_{U V}\left(a_{j} ; \mathbf{t}, T\right)+\sum_{i \neq j} F\left(a_{i}, a_{j} ; T\right) \\
a_{j}=T\left(v_{j}\right), \quad j=1, \ldots, N_{c} \\
F_{U V}(a ; \mathbf{t}, \mathbf{T})=F_{U V}(v(a) ; \mathbf{t}, \mathbf{T})=\int_{0}^{v(a)} \mathbf{t}^{\prime}(\mathbf{v}) d T(\mathbf{v})=\int_{0}^{a} \mathbf{t}^{\prime}(\mathbf{v}(\mathbf{a})) d \mathbf{a} \\
\frac{\partial^{2}}{\partial a_{i} \partial a_{j}} F\left(a_{i}, a_{j} ; \mathbf{T}\right)=\log \left(v_{i}\left(a_{i}, \mathbf{T}\right)-v_{j}\left(a_{j}, \mathbf{T}\right)\right)
\end{gathered}
$$

with $v(a)=v(a, T)=T^{-1}(a)$, being a solution with asymptotic $v=\frac{a}{T_{1}}+\ldots$ for small higher times.

Before considering the nonperturbative formulation on smooth curve (4.1) it is instructive to discuss the relation of already obtained in $N_{c}=1$ case formulas with the functional formulation. As in the half-truncated theory $[2,12]$ we postulate, that the linear and bilinear parts of the functional are directly determined by the perturbative prepotential (4.26). In its turn, the functional formulation would become a good "reference point" for the construction in terms of abelian differentials on smooth hyperelliptic curve (4.1). 


\section{Functional methods and Virasoro constraints}

Let us now turn to the functional formulation of the proposed above analytic formulas. To remind, we start first with the case, when all gravitational descendants of unity are switched off, except for the condensate of $\left\langle\sigma_{1}(\mathbf{1})\right\rangle \neq 0$.

Switched off $T_{n}, n>1$

The curve (2.1) endowed with the function (2.4) arises [2] in the extremum problem for the functional

$$
\mathcal{F}=\frac{1}{2} \int d x f^{\prime \prime}(x) \mathbf{t}(x)-\frac{1}{2} \int_{x_{1}>x_{2}} d x_{1} d x_{2} f^{\prime \prime}\left(x_{1}\right) f^{\prime \prime}\left(x_{2}\right) F\left(x_{1}-x_{2}\right)
$$

extremized w.r.t. second derivative of the profile function $f^{\prime \prime}(x)=\frac{d^{2} f}{d x^{2}}$, constrained by

$$
1=\frac{1}{2} \int d x f^{\prime \prime}(x)=\left.\frac{1}{2} f^{\prime}(x)\right|_{x_{-}} ^{x_{+}}
$$

together with

$$
T_{0}=-a=-\frac{1}{2} \int d x x f^{\prime \prime}(x)=\left.\frac{1}{2}\left(f(x)-x f^{\prime}(x)\right)\right|_{x_{-}} ^{x_{+}}
$$

and where the kernel is

$$
F(x)=\frac{1}{2} H_{2}^{(+)}(x)=\frac{x^{2}}{2}\left(\log x-\frac{3}{2}\right)
$$

while the source $\mathbf{t}(x)$ is defined by ultraviolet prepotential in (1.1).

Constraints (5.2), (5.3) can be taken into account by adding them to the functional (5.1) with the Lagrange multipliers

$$
\mathcal{F} \rightarrow \mathcal{F}+a^{D}\left(a-\frac{1}{2} \int d x x f^{\prime \prime}(x)\right)+\sigma\left(1-\frac{1}{2} \int d x f^{\prime \prime}(x)\right)
$$

so that the variational equation for (5.5) reads

$$
\mathbf{t}(x)-\int d \tilde{x} f^{\prime \prime}(\tilde{x}) F(x-\tilde{x})=a^{D} x+\sigma
$$

One also gets from (5.1)

$$
\frac{\partial \mathcal{F}}{\partial t_{k}}=\frac{1}{2(k+1)} \int d x f^{\prime \prime}(x) x^{k+1}, \quad k>0
$$


and, due to (5.5)

$$
a^{D}=\frac{\partial \mathcal{F}}{\partial a}
$$

The second Lagrange multiplier in (5.5)

$$
\sigma=-\left(S_{1}\right)_{0}=\frac{\partial \mathcal{F}}{\partial T_{1}}
$$

is given by the derivative of prepotential w.r.t. the first flow of the extended hierarchy. We remind that the derivatives over the Lagrange multipliers can be taken directly, at constant $f^{\prime \prime}(x)$, since all other contributions to these derivatives are proportional to the extremum equation, and therefore vanish on its solutions.

Integrating (5.6), one gets the double-integral representation

$$
\mathcal{F}=\frac{1}{2} \int_{x_{1}>x_{2}} d x_{1} d x_{2} f^{\prime \prime}\left(x_{1}\right) f^{\prime \prime}\left(x_{2}\right) F\left(x_{1}-x_{2}\right)+a a^{D}+\sigma
$$

which, together with (5.1), gives

$$
\mathcal{F}=\frac{1}{4} a a^{D}+\frac{1}{2} \sigma+\frac{1}{4} \int d x f^{\prime \prime}(x) \mathbf{t}(x)=\frac{1}{4} a \frac{\partial \mathcal{F}}{\partial a}+\frac{1}{2} \sum_{k>0} t_{k} \frac{\partial \mathcal{F}}{\partial t_{k}}+\frac{1}{2} \sigma
$$

where the last equality follows from (5.7), (5.8). Comparing it with representation

$$
\mathcal{F}=\frac{1}{2}\left(a \frac{\partial \mathcal{F}}{\partial a}+\sum_{k>1}(1-k) t_{k} \frac{\partial \mathcal{F}}{\partial t_{k}}\right)+\frac{\partial \mathcal{F}}{\partial t_{1}}-\frac{a^{2}}{2}
$$

and using (5.9), one derives

$$
\frac{\partial \mathcal{F}}{\partial T_{1}}+\frac{1}{2} a \frac{\partial \mathcal{F}}{\partial a}-\sum_{k>0} k t_{k} \frac{\partial \mathcal{F}}{\partial t_{k}}+2 \frac{\partial \mathcal{F}}{\partial t_{1}}-a^{2}=0
$$

or the quasiclassical $L_{0}$-Virasoro constraint at fixed $T_{n}=\delta_{n, 1}$.

\section{Quasiclassical Virasoro constraints}

The following integral along the boundary of the cut cylinder

$$
\oint \Phi^{2} z^{n+1} d z=\oint\left(\frac{d S}{d z}\right)^{2} z^{n+1} d z=0, \quad n=-1,0,1,2, \ldots
$$


vanishes, since, analogously to [4],

$$
\Phi=\frac{d S}{d z} \underset{z \rightarrow \infty}{=}-2 \sum_{n>0} n T_{n} z^{n-1}\left(\log z-c_{n-1}\right)+\sum_{k>0} k t_{k} z^{k-1}+\frac{2 a}{z}+2 \sum_{k=1}^{\infty} \frac{1}{z^{k+1}} \frac{\partial \mathcal{F}}{\partial t_{k}}
$$

has no singularities in the interior of the cut cylinder, since $d S=0$ at the branching points, where $d z=0$.

Technically, it is simpler instead of (5.14) to consider the "string equations", or the $a$ derivative of this formula. Namely,

$$
\oint \Phi z^{n+1} \frac{\partial}{\partial a} \Phi d z=\oint \Phi z^{n+1} \frac{d w}{w}=0
$$

since all time-derivatives are taken at constant $z$. Moreover, one can take care only of the constant part of the contributions into (5.14) and (5.16) from the $A$-and $B$-integrals, forming the boundary of the cut cylinder, see details in Appendix. For example, if $n=-1$ and only $T_{1} \neq 0$, formula $(5.16)$ gets two obvious contributions

$$
\left[\int_{B^{+}}+\int_{B^{-}}\right] \Phi \frac{d w}{w} \sim T_{1} \int_{B} \frac{d w}{w} \sim T_{1} \frac{\partial^{2} \mathcal{F}}{\partial a^{2}}
$$

while

$$
\left[\int_{A^{+}}+\int_{A^{-}}\right] \Phi \frac{d w}{w} \sim \operatorname{res}_{\infty}\left(\mathbf{t}^{\prime \prime}(z) \frac{d w}{w}\right) \sim t_{1}+\sum_{k>1} k t_{k} \frac{\partial^{2} \mathcal{F}}{\partial a \partial t_{k-1}}
$$

which form together the desired string equation, or $a$-derivative of the $L_{-1}$ Virasoro constraint from $[6,9]$.

\section{Functional with all descendants switched on}

The perturbative formulas in the nonabelian case (4.26) suggest the following form of the functional with all gravitational descendants stitched on

$$
\begin{gathered}
\mathcal{F}=\frac{1}{2} \int d x f^{\prime \prime}(x) F_{U V}(x)+\frac{1}{2} \int_{x_{1}>x_{2}} d x_{1} d x_{2} f^{\prime \prime}\left(x_{1}\right) f^{\prime \prime}\left(x_{2}\right) F\left(x_{1}, x_{2} ; \mathbf{T}\right)+ \\
+a^{D}\left(a-\frac{1}{2} \int d x f^{\prime \prime}(x) T(x)\right)+\sigma\left(1-\frac{1}{2} \int d x f^{\prime \prime}(x)\right)
\end{gathered}
$$

where $^{1}$

$$
\begin{gathered}
F_{U V}(x) \equiv F_{U V}(x ; \mathbf{t}, \mathbf{T})=\int_{0}^{x} \mathbf{t}^{\prime}(\mathbf{x}) d T(\mathbf{x}) \\
\frac{\partial^{2}}{\partial x_{1} \partial x_{2}} F\left(x_{1}, x_{2} ; \mathbf{T}\right)=T^{\prime}\left(x_{1}\right) T^{\prime}\left(x_{2}\right) \log \left(x_{1}-x_{2}\right)
\end{gathered}
$$

\footnotetext{
${ }^{1}$ Formulas (5.19) and (5.20) were derived earlier by N. Nekrasov, in a similar context, but using different arguments.
} 
The variation of (5.19) over $f^{\prime \prime}(x)$ gives

$$
F_{U V}(z)+\int d x f^{\prime \prime}(x) F(z, x ; T)=a^{D} T(z)+\sigma, \quad z \in \mathbf{I}
$$

whose $z$-derivative, after dividing by $T^{\prime}(z)$, turns into

$$
\mathbf{t}^{\prime}(z)-\int d x f^{\prime \prime}(x) \sigma(z ; x)=a^{D}, \quad z \in \mathbf{I}
$$

Due to the property of the function $\sigma(z, x)$, following directly from its definition (4.22) and expansion

$$
\begin{gathered}
\frac{1}{n !} H_{n}^{(+)}(z-x)=\frac{1}{n !}(z-x)^{n}\left(\log (z-x)-c_{n}\right)= \\
=\sum_{k=0}^{n} \frac{H_{n-k}^{(+)}(z)}{(n-k) !} \frac{(-x)^{k}}{k !}+(-)^{n-1} \sum_{k>0} \frac{x^{n+k}}{k z^{k}} \frac{1}{(k+1) \ldots(k+n)}
\end{gathered}
$$

one gets (for the switched on $N$ descendants of unity)

$$
\sigma(z ; x)=\sum_{k>0} \frac{T^{(k)}(x)}{k !} H_{k}^{(+)}(z-x)=\sum_{n=1}^{N} T_{n} H_{n}^{(+)}(z)-T(x) \log z+\sum_{k>0} \frac{F_{k}(x)}{k z^{k}}
$$

where

$$
F_{k}(x)=\int_{0}^{x} \mathrm{x}^{k} d T(\mathrm{x})
$$

and we have used the obvious polynomial identities

$$
k ! T_{k}=T^{(k)}(0)=\sum_{n=0}^{N} \frac{T^{(n+k)}(x)}{n !}(-x)^{n}, \quad k=0, \ldots, N
$$

From (5.22) it follows, that the integral

$$
\begin{gathered}
S(z)=\mathbf{t}^{\prime}(z)-\int d x f^{\prime \prime}(x) \sigma(z ; x)-a^{D}= \\
=\mathbf{t}^{\prime}(z)-a^{D}-\sum_{k>0} \int d x f^{\prime \prime}(x) \frac{T^{(k)}(x)}{k !} H_{k}^{(+)}(z-x)
\end{gathered}
$$

whose real part vanishes on the support by (5.22) has an asymptotic expansion (3.15) and is constant on the cut. Moreover, the coefficients at negative powers of $z$ in the r.h.s. are given by

$$
\int d x f^{\prime \prime}(x) F_{k}(x)=2 \int d x f^{\prime \prime}(x) \frac{\partial F_{U V}(x ; \mathbf{t}, \mathbf{T})}{\partial t_{k}}=2 \frac{\partial \mathcal{F}}{\partial t_{k}}
$$


However, it is not easy to get any simple expression for the $T_{n}$-derivatives of the functional (5.19), since almost everything depends on $\left\{T_{n}\right\}$ in the r.h.s. of this formula. In order to get the new formula (3.12) for the derivatives over the variables extending the Toda chain hierarchy, one has to consider a different form of the functional (5.19).

\section{Another form of the functional}

The formula (5.5) in fact suggests how the functional problem can be re-formulated in a different way, when the higher times of extended hierarchy are switched of. Suppose again that only $T_{1}, \ldots, T_{N}$ are non-vanishing, which somehow characterize the $N$-th "class of backgrounds" for the gauge theory. One can write for the perturbative prepotential

$$
\begin{gathered}
F_{U V}(x)=\int_{0}^{x} \mathbf{t}^{\prime}(\mathbf{x}) d T(\mathbf{x})=\mathbf{t}(x) T^{\prime}(x)-\mathbf{t}_{2}(x) T^{\prime \prime}(x)+\ldots+(-)^{N-1} \mathbf{t}_{N}(x) T^{(N)}= \\
=\hat{D}_{N-1}(x) \mathbf{t}_{N}(x)
\end{gathered}
$$

where

$$
\begin{aligned}
\mathbf{t}_{N}(x) & =\sum_{k>0} t_{k} \frac{x^{k+N}}{(k+1) \ldots(k+N)} \\
\mathbf{t}(x) & \equiv \mathbf{t}_{1}(x)=\frac{d^{N-1}}{d x^{N-1}} \mathbf{t}_{N}(x)
\end{aligned}
$$

and we have introduced the differential operator with the polynomial coefficients

$$
\hat{D}_{N-1}(x)=T^{\prime}(x) \frac{d^{N-1}}{d x^{N-1}}-T^{\prime \prime}(x) \frac{d^{N-2}}{d x^{N-2}}+\ldots+(-)^{N-1} T^{(N)}
$$

Consider also an integral transform, or introduce new "density" by the formula

$$
\int d x \rho(x) g(x)=\int d x f^{\prime \prime}(x) \hat{D}_{N-1}(x) g(x)
$$

for an integral over the support I with an arbitrary function $g(x)$ (from some reasonable class of functions). It means, that in certain sense this density is $\rho(x) \sim \hat{D}_{N-1}^{\dagger}(x) f^{\prime \prime}(x)$. Note also, that using $\hat{D}$-operators (5.29), one can write for the kernel in (5.20)

$$
\begin{gathered}
F(z, x)=\frac{(-)^{N}}{(2 N) !} \hat{D}_{N-1}(z) \hat{D}_{N-1}(x) H_{2 N}^{(+)}(z-x)= \\
=\sum_{n, k=1}^{N}(-)^{n-1} T^{(n)}(z) T^{(k)}(x) \frac{H_{n+k}^{(+)}(z-x)}{(n+k) !}
\end{gathered}
$$

while the contribution of the linear term in (5.19) - with the ultraviolet prepotential - turns into

$$
\int d x f^{\prime \prime}(x) F_{U V}(x)=\int d x \rho(x) \mathbf{t}_{N}(x)
$$


The density $\rho(x)$ obeys important constraints, directly following from (5.32), namely

$$
\begin{gathered}
\frac{1}{2} \int d x \rho(x) \frac{x^{n}}{n !}=\frac{1}{2} \int d x f^{\prime \prime}(x) \hat{D}_{N-1}(x) \frac{x^{n}}{n !}=(-)^{n-1} T_{N-n} \\
n=0,1,2, \ldots, \quad T_{0}=-a
\end{gathered}
$$

which have to be taken into account, if one considers variation of the functional over the new density.

In other words, instead of (5.1) one can consider an extremum for

$$
\begin{aligned}
\mathcal{F}=\mathcal{F}_{N}[\rho]=\frac{1}{2} \int & d x \rho(x) \mathbf{t}_{N}(x)-\frac{(-)^{N-1}}{2(2 N) !} \int_{x_{1}>x_{2}} d x_{1} d x_{2} \rho\left(x_{1}\right) \rho\left(x_{2}\right) H_{2 N}^{(+)}\left(x_{1}-x_{2}\right)+ \\
& +\sum_{n=0}^{N} \sigma_{n}\left(T_{n}-\frac{(-)^{n-1}}{2} \int d x \frac{x^{N-n}}{(N-n) !} \rho(x)\right)
\end{aligned}
$$

where the kernel $\frac{(-)^{N-1}}{(2 N) !} H_{2 N}^{(+)}(x)=\frac{1}{(2 N) !} x^{2 N}\left(\log x-c_{2 N}\right)$ does not depend explicitly of the times $\mathbf{T}$, all this dependence is absorbed by density $\rho(x)$. The extremum condition for the functional (5.36) stays, that (real part of)

$$
S_{N}(z)=\mathbf{t}_{N}(z)-\frac{(-)^{N-1}}{(2 N) !} \int d x \rho(x) H_{2 N}^{(+)}(z-x)+\sum_{n=0}^{N} \sigma_{n}(-)^{n} \frac{z^{N-n}}{(N-n) !}
$$

vanishes on the support $z \in \mathbf{I}$ of $\rho(z)$. Taking up to $N$-th derivatives of (5.37) one gets

$$
\begin{gathered}
S_{N-1}(z)=\mathbf{t}_{N-1}(z)-\frac{(-)^{N-1}}{(2 N-1) !} \int d x \rho(x) H_{2 N-1}^{(+)}(z-x)+\sum_{n=0}^{N-1} \sigma_{n}(-)^{n} \frac{z^{N-n-1}}{(N-n-1) !} \\
\vdots \\
S(z)=\mathbf{t}^{\prime}(z)-\frac{(-)^{N-1}}{N !} \int d x \rho(x) H_{N}^{(+)}(z-x)+\sigma_{0}
\end{gathered}
$$

a sequence of functions vanishing on the cut. The last integral $S(z)=\frac{d^{N}}{d z^{N}} S_{N}(z)$ coincides with (5.27), and therefore has the same properties.

In particular, at $z \rightarrow \infty$ the last integral in (5.38) has an expansion where the coefficients are expressed by the "moments" of new density

$$
\begin{aligned}
& S(z) \underset{z \rightarrow \infty}{=} \mathbf{t}^{\prime}(z)-\sum_{n=0}^{N} z^{n}\left(\log z-c_{n}\right) \frac{(-)^{N-n}}{(N-n) !} \int x^{N-n} \rho(x) d x+\sigma_{0}+ \\
& -\sum_{k>0} \frac{1}{k(k+1) \ldots(k+N) z^{k}} \int x^{N+k} \rho(x) d x= \\
& =\mathbf{t}^{\prime}(z)-2 \sum_{n=0}^{N} T_{n} z^{n}\left(\log z-c_{n}\right)-\frac{\partial \mathcal{F}}{\partial a}-2 \sum_{k>0} \frac{1}{k z^{k}} \frac{\partial \mathcal{F}}{\partial t_{k}}
\end{aligned}
$$


reproducing (3.15) by (5.32) and (5.35) (or upon the constraints at Lagrange multipliers in (5.36)). From the properties of the functional (5.36), one can straightforwardly find the derivatives

$$
\begin{gathered}
\frac{\partial \mathcal{F}}{\partial t_{k}}=\frac{1}{2(k+1) \ldots(k+N)} \int x^{k+N} \rho(x) d x \\
\sigma_{0}=\frac{\partial \mathcal{F}}{\partial T_{0}}=-\frac{\partial \mathcal{F}}{\partial a}=-a^{D}
\end{gathered}
$$

coinciding with (5.28).

However, after arbsorbing all nontrivial T-dependence into $\rho$ in (5.36), it becomes obvious, that

$$
\frac{\partial \mathcal{F}}{\partial T_{n}}=\sigma_{n}=(-)^{n} n !\left(S_{n}\right)_{0}, \quad n=0, \ldots, N
$$

The naively divergent integrals, containing $\rho(x)$, should be understood only in the sense of $(5.32)$.

\section{$6 \quad$ Nonabelian theory from abelian integrals}

Finally, let us turn to discussion of generic nonabelian theory, whose perturbative limit was considered in sect. 4. The quasiclassical tau-function is now defined by constructing an abelian integral on the hyperelliptic curve (4.1), whose properties can be extracted from integral representations of sect. 5 .

It is again important to fix certain finite number $N$ of the gravitational descendants of unity being switched on. The integral representation (5.27) defines a multivalued abelian integral on the curve (4.1), and only its $N$-th derivative becomes single-valued. Denote as usual $\Phi=\frac{d S}{d z}$, and further $\Phi^{\prime}=\frac{d \Phi}{d z}, \ldots$ up to

$$
\begin{gathered}
d \Phi^{(N-1)}=d\left(\frac{d^{N-1} \Phi}{d z^{N-1}}\right)= \\
=\mathbf{t}^{(N+2)}(z) d z-N ! T_{N} \int \frac{f^{\prime \prime}(x) d x}{z-x} d z-\sum_{k=1}^{N-1}(-)^{k} \int \frac{T^{(N-k)}(x) f^{\prime \prime}(x) d x}{(z-x)^{k+1}} d z
\end{gathered}
$$

which is already a single-valued on the non-degenerate curve (4.1) abelian differential, odd under the hyperelliptic involution, since its real part vanishes on the cut. Its form can be totally determined by its singularities at the infinity points $P_{ \pm}$and at the ramification points $\left\{x_{j}\right\}, j=1, \ldots, 2 N_{c}$, where it also has poles due to behavior $f^{\prime \prime}(x) \sim\left(x-x_{ \pm}\right)^{-1 / 2}$. The singularities at ramification points are in fact artificial, in the sense that one may think of $\Phi^{\prime}, \ldots, \Phi^{(N-1)}$ as of the regular at branch points $2-, \ldots, N$ - differentials on the curve (4.1). 
One can therefore write for (6.1) an explicit formula

$$
d \Phi^{(N-1)}=\frac{\phi(z) d z}{y}+\frac{d z}{y} \sum_{j=1}^{2 N_{c}} \sum_{k=1}^{N-1}\left(\frac{q_{j}^{k}}{\left(z-x_{j}\right)^{k}}\right)
$$

where $\phi(z)$ is a polynomial of power

$$
\operatorname{deg} \phi(z)=\left\{\begin{array}{c}
N_{c}-1, \quad n \leq N \\
N_{c}-1+n-N, \quad n>N
\end{array}\right.
$$

for the theory on genus $N_{c}-1$ curve (4.1) and with $n-1$ and $N$ nonvanishing times $\left\{t_{k}\right\}$ and $\left\{T_{n}\right\}$ correspondingly. The periods of (6.2) are fixed by (6.1), or

$$
\begin{gathered}
\frac{1}{2 \pi i} \oint_{A_{i}} d \Phi^{(N-1)}=-2 N ! T_{N} \\
\oint_{B_{i}} d \Phi^{(N-1)}=0
\end{gathered}
$$

Couning the period constraints (6.4), one can consider $N_{c}$ cycles $A_{k}, k=1, \ldots, N_{c}$, surrounding generally $N_{c}$ distinct segments of the support of $f^{\prime \prime}(x) \neq 0, x \in \mathbf{I}_{k}, k=1, \ldots, N_{c}$, which is equivalent to the canonical choice of $A$-cycles together with the residue at infinity. Totally, (6.4) give $2 N_{c}-1$ period constraints, and should be completed by the $2 N_{c}$-th condition

$$
\int_{z\left(P_{-}\right)}^{z\left(P_{+}\right)} d \Phi^{(N-1)}=-2 N ! N_{c} T_{N} \log z+4 \pi i N ! T_{N} \mathbb{Z}+O\left(\frac{1}{z}\right)
$$

i.e. the regularized constant part of the integral $\int_{P_{-}}^{P_{+}} d \Phi^{(N-1)}$ vanishes modulo the period lattice (6.4), since the integral (6.5) depends on the choice of the integration path.

Small phase space and $T_{2} \neq 0$

Consider for simplicity only $t_{1} \neq 0$ and switched on $T_{1}, T_{2}$. Formula (6.1) gives for this case

$$
d \Phi^{\prime}=\frac{\phi_{N_{c}-1}(z) d z}{y}+\frac{d z}{y} \sum_{j=1}^{2 N_{c}}\left(\frac{q_{j}}{z-x_{j}}\right)
$$

which depends on $3 N_{c}$ coefficients of $\phi_{N_{c}-1}(z)$ and $\left\{q_{j}\right\}$, as well as $2 N_{c}$ branch points $\left\{x_{j}\right\}$, i.e. totally of $5 N_{c}$ undetermined yet coefficients. The period integrals (6.4), together with the residue

$$
\operatorname{res}_{P_{+}} d \Phi^{\prime}=-\operatorname{res}_{P_{-}} d \Phi^{\prime}=4 T_{2} \cdot N_{c}
$$


give altogether $2 N_{c}$ constraints, or fix the parameters $\left\{q_{j}\right\}$ of the differential (6.6), leaving yet no restrictions for the coefficients of $\phi_{N_{c}-1}(z)$ and branch points of the curve.

Now, one can define an abelian integral $\Phi^{\prime}(P)=\int^{P} d \Phi^{\prime}$ or the differential

$$
d \Phi=d z \int^{z} d \Phi^{\prime}
$$

which is multivalued, but all the jumps are fixed by (6.4), being proportional to $4 \pi i \cdot T_{2} d z$. The integration constant in (6.8) is fixed by requirement, that $\Phi^{\prime}(P) \underset{z(P) \rightarrow \infty}{\sim}-4 N_{c} T_{2} \log z+O\left(\frac{1}{z}\right)$, consistent due to (6.5). Since the differential of hyperelliptic co-ordinate on (4.1) has vanishing periods $\oint d z=0$ along any cycle, one can make sense of the periods of the differential (6.8) itself, and put

$$
\begin{gathered}
\frac{1}{2 \pi i} \oint_{A_{k}} d \Phi \equiv-\frac{1}{2 \pi i} \oint_{A_{k}} z d \Phi^{\prime}=T_{1} \int_{\mathbf{I}_{k}} d x f^{\prime \prime}(x)=2 T_{1}, \quad k=1, \ldots, N_{c} \\
\oint_{B_{k}} d \Phi \equiv \oint_{B_{k}} z d \Phi^{\prime}=0
\end{gathered}
$$

The period integrals (6.9) together with normalization condition (say, $\Phi\left(x_{N_{c}}\right)=0$ ) give $2 N_{c}$ more constraints on the total set of undetermined parameters, while the rest is absorbed by the Seiberg-Witten periods, defined now as

$$
a_{j}=\frac{1}{4 \pi i} \oint_{A_{j}} \frac{z^{2}}{2} d \Phi^{\prime}, \quad j=1, \ldots, N_{c}
$$

whose sum gives the residue at infinity.

\section{$N$ descendants $T_{1}, \ldots, T_{N} \neq 0$}

Almost the same counting can be performed for the generic case with $N$ descendants. One has now $2 N_{c} \cdot N+N_{c}=(2 N+1) N_{c}$ parameters of $\phi_{N_{c}-1}(z),\left\{q_{j}^{k}\right\}$ and branch points $\left\{x_{j}\right\}$ (in the case of nonvanishing higher $\left\{t_{k}\right\}$ they will be absorbed into higher coefficients of the polynomial $\phi(z)$ and the integration constants). Being constrained by constancy of its periods, we rest with $(2 N-1) N_{c}$ variables.

We have then to restore the differential $d \Phi$ by multiple integration of (6.1). At each step we have to fix the periods of $d \Phi^{(N-2)}, \ldots, d \Phi^{\prime}$ by $2 N_{c}$ constraints, ending up, therefore with

$$
(2 N+1) N_{c}-2 N_{c} \cdot N=N_{c}
$$

variables, which can be conveniently chosen as the Seiberg-Witten periods

$$
a_{j}=\frac{1}{4 \pi i} \oint_{A_{j}} \frac{z^{N}}{N !} d \Phi^{(N-1)}, \quad j=1, \ldots, N_{c}
$$


The multivalued differential $d S=\Phi d z$ has now constant jumps, depending linearly upon $a$ and the times $T_{1}, \ldots, T_{N}$, and one can always choose its branch with the asymptotic (3.15), if taken along the real axis at $z \rightarrow+\infty$ on the "upper" sheet.

\section{Quasiclassical tau-function}

The dual periods

$$
a_{j}^{D}=\frac{1}{2} \oint_{B_{j}} \frac{z^{N}}{N !} d \Phi^{(N-1)}=\frac{\partial \mathcal{F}}{\partial a_{j}}, \quad j=1, \ldots, N_{c}
$$

define the gradients of the quasiclassical tau-function. Integrability condition for (6.13) is guaranteed by symmetricity of the period matrix of the curve (4.1), following from

$$
\delta(d S)=\delta(\Phi d z) \simeq \text { holomorphic }
$$

following directly from the constancy of the periods $d \Phi^{\prime}, \ldots, d \Phi^{(N-1)}$. In addition to the remaining intact "abelian formulas" (3.14) and (3.12) that defines the full quasiclassical tau-function for the perturbed theory, and the integrability is guaranteed by the Riemann bilinear relations.

\section{Discussion}

We have presented in this paper a quasiclassical geometric formulation for the full non-truncated topological $\mathbb{P}^{1}$ string model, when all the descendants $\sigma_{k}(\varpi)$ and $\sigma_{k}(\mathbf{1})$ with $k>0$ are switched on, and propose its generalization to the nonabelian dual supersymmetric gauge theory. For the topological string model the quasiclassical formulation is given in "mirror" terms - a rational curve, which can be interpreted as a dual Landau-Ginzburg superpotential $z=v+\Lambda\left(w+\frac{1}{w}\right)$ on a cylinder, and the set of functions, odd under its involution $w \leftrightarrow \frac{1}{w}$. The descendants of the Kähler class $\sigma_{k}(\varpi)$ generate the flows of dispersionless Toda chain hierarchy, while the descendants of unity $\sigma_{k}(\mathbf{1})$ produce the logarithmic flows [5] of the so called [10] extended Toda hierarchy, which can be possibly reformulated as a reduction of two-dimensional Toda lattice. The exact relation of the quasiclassical solution, proposed above, to the two-dimensional Toda lattice is beyond the scope of this paper, but let us present here a hint, how the multiple integral formula (3.12) can be interpreted in this way.

\section{Equivariant Toda lattice}

The relation between the extended Toda and equivariant Toda lattice $[8,11]$ includes the change of the variables

$$
X_{k+1}=\frac{T_{k}}{\epsilon}+t_{k+1}, \quad \bar{X}_{k+1}=-\frac{T_{k}}{\epsilon}, \quad k \geq 0
$$


or

$$
\begin{gathered}
t_{k}=X_{k}+\bar{X}_{k}, \quad k>0 \\
T_{k}=\epsilon\left(X_{k+1}-\bar{X}_{k+1}\right), \quad k \geq 0
\end{gathered}
$$

For example, the prepotential on the small phase space

$$
\begin{gathered}
\mathcal{F}\left(X_{1}, \bar{X}_{1} ; \epsilon\right)=\frac{\epsilon^{2}}{6}\left(X_{1}^{3}+\bar{X}_{1}^{3}\right)+e^{X_{1}+\bar{X}_{1}}= \\
=\frac{a^{2} t_{1}}{2}+e_{1}^{t}+\frac{\epsilon}{2} a t_{1}^{2}+\frac{\epsilon^{2}}{6} t_{1}^{3}
\end{gathered}
$$

coinciding with (2.18) at $\epsilon \rightarrow 0$, indeed satisfies the two-dimensional Toda lattice equation

$$
\frac{\partial^{2} \mathcal{F}}{\partial \bar{X}_{1} \partial X_{1}}=\exp \left(\frac{1}{\epsilon^{2}}\left(\frac{\partial}{\partial X_{1}}-\frac{\partial}{\partial \bar{X}_{1}}\right)^{2} \mathcal{F}\right)
$$

if one takes the solutions, constrained by reduction, including

$$
\frac{\partial \mathcal{F}}{\partial X_{1}}-\frac{\partial \mathcal{F}}{\partial \bar{X}_{1}}=\epsilon \frac{\partial \mathcal{F}}{\partial X_{0}}
$$

One can expect therefore, generally, that

$$
\frac{\partial \mathcal{F}}{\partial X_{k}}-\frac{\partial \mathcal{F}}{\partial \bar{X}_{k}}=\epsilon \hat{R}_{k} \circ \mathcal{F}, \quad \forall k>0
$$

where $\hat{R}_{k}$ is presumably a $\left(k\right.$-th order) differential operator in $X_{0}, \hat{R}_{1}=\frac{\partial}{\partial X_{0}}$. At $\epsilon \rightarrow 0$ conditions (7.6), (7.5) turn into the Toda chain reduction

$$
\begin{gathered}
\frac{\partial \mathcal{F}}{\partial X_{k}}-\frac{\partial \mathcal{F}}{\partial \bar{X}_{k}}=0, \quad \forall k>0 \\
\frac{\partial \mathcal{F}}{\partial X_{k}}+\frac{\partial \mathcal{F}}{\partial \bar{X}_{k}}=2 \frac{\partial \mathcal{F}}{\partial t_{k}}, \quad \forall k>0
\end{gathered}
$$

where $\left\{t_{k}\right\}$ (7.2) are the times of the Toda chain. More generally, in the reduction to the Toda chain, the first set of conditions (7.7) can have a linear function at the r.h.s.

$$
\frac{\partial \mathcal{F}}{\partial X_{k}}-\frac{\partial \mathcal{F}}{\partial \bar{X}_{k}}=C_{k}\left(X_{k}-\bar{X}_{k}\right), \quad \forall k>0
$$

with $C_{k} \sim k$ is a constant as a function of times. For the function (7.3) one gets instead if (7.8)

$$
\frac{\partial \mathcal{F}}{\partial X_{1}}-\frac{\partial \mathcal{F}}{\partial \bar{X}_{1}}=\frac{\epsilon}{2}\left(X_{1}^{2}-\bar{X}_{1}^{2}\right)=\frac{\epsilon}{2}\left(X_{1}+\bar{X}_{1}\right)\left(X_{1}-\bar{X}_{1}\right)
$$

so one finds, that $C_{1}=\frac{\epsilon}{2}\left(X_{1}+\bar{X}_{1}\right)=\frac{\epsilon}{2} t_{1}$ instead of a constant becomes a "slow" modulated linear function of the Toda chain time $t_{1}$. The exact form of the operators $\hat{R}_{k}$ is not yet 
known (though perhaps can be extracted from [8]), but the formulas (3.12), (A.1) establish the quasiclassical correspondence

$$
\hat{R}_{n} \mathcal{F} \sim[\underbrace{\int_{d z} \ldots \int_{d z}}_{n} S]_{0}
$$

For the two-dimensional Toda lattice one has two different co-ordinates $z_{+}$and $z_{-}$at two infinities $P_{ \pm}$corresponding to the flows in $X$ and $\bar{X}$ time variables. One may think then, that $z_{+}-z_{-} \sim \int d S$ and the differences of the higher Hamiltonians $\Omega\left(z_{+}\right)-\tilde{\Omega}\left(z_{-}\right) \sim \int \ldots \int d S$ produces the desired formula (7.10).

\section{Nonabelian theory}

It is not yet completely clear, when is the sense of "descendant" deformation of the nonabelian theory. The descendants of the Kähler class deform the gauge theory in the ultraviolet, which is encoded in $\frac{1}{2} \tau_{0} x^{2} \rightarrow F_{U V}(x ; \mathbf{t})$ for the short-distance prepotential (1.1). The descendants of unity perform rather a reparameterization on the moduli space of gauge theory $a_{j} \rightarrow T\left(a_{j}\right)+$ $O\left(\Lambda^{2 N_{c}}\right)$, whose exact sense remains yet unclear.

We have considered in $[2,12]$ and above here the theory, where all $t_{k}$ with $k>1$ and $T_{n}$ with $n>1$ generate infinitesimal perturbations of the model on "small phase space". Nevertheless, all descendants deform the Seiberg-Witten curve (except for the "abelian" case of the $\mathbb{P}^{1}$ model), which now turns to be a generic hyperelliptic curve (4.1), though still being "not to far" in the moduli space from the Seiberg-Witten curve (4.2). In particular, we do not address any questions, related with possible "large" deformations in moduli space, changing the genus etc. Roughly speaking, if the t-deformations of the theory lead us towards the processes of generation of fundamental multiples, in the same sense the $T$-deformations lead towards embedding of the theory into the compactified higher-dimensional target spaces.

\section{Acknowledgements}

I am grateful to A. Alexandrov, M. Kazaryan, S. Kharchev, I. Krichever, A. S. Losev, A. Mironov, and N. Nekrasov for the very useful discussions. I would like also to thank Institut des Hautes Etudes Scientifiques at Bures-sur-Yvette and Laboratoire de Mathematiques et Physique Theorique at Universite Francois Rabelais, Tours, where essential parts of this work have been done, for warm hospitality.

The work was partially supported by the Federal Nuclear Energy Agency, the RFBR grant 06-02-17383 the grant for support of Scientific Schools 4401.2006.2, INTAS grant 05-10000087865, the project ANR-05-BLAN-0029-01, the NWO-RFBR program 047.017.2004.015, the Russian-Italian RFBR program 06-01-92059-CE, and by the Dynasty foundation. 


\section{Appendix}

\section{A Riemann bilinear identities}

Equation (3.12), or

$$
\left.\frac{\partial \mathcal{F}}{\partial T_{n}}\right|_{\mathbf{t}}=[\underbrace{\int_{d z} \cdots \int_{d z}}_{n} S]_{0}
$$

gives rise to the mixed second derivatives

$$
\frac{\partial^{2} \mathcal{F}}{\partial t_{k} \partial T_{n}}=[\underbrace{\int_{d z} \ldots \int_{d z}}_{n} \Omega_{k}]_{0}
$$

which should be compared to

$$
\frac{\partial^{2} \mathcal{F}}{\partial T_{n} \partial t_{k}}=\frac{1}{2} \operatorname{res}_{P_{+}} z^{k} d H_{n}=-\frac{1}{2} \operatorname{res}_{P_{-}} z^{k} d H_{n},
$$

following from (2.8). In order to establish equivalence between (A.2) and (A.3), consider the integral along the boundary of the cut $w$-cylinder with the removed points $P_{ \pm}$

$$
\oint_{\partial \Sigma} H_{n} d \Omega_{k}=2 \pi i \sum \operatorname{res} H_{n} d \Omega_{k}=0
$$

The integral in the l.h.s. can be rewritten as

$$
\oint_{\partial \Sigma} H_{n} d \Omega_{k}=\left[\int_{A^{+}}+\int_{A^{-}}\right] H_{n} d \Omega_{k}+\left[\int_{B^{+}}+\int_{B^{-}}\right] H_{n} d \Omega_{k}
$$

where we have chosen the following parameterization of the cut $w$-cylinder:

$$
\begin{gathered}
A^{+}: \quad w=\varepsilon e^{i \varphi}, \quad 0<\varphi<2 \pi \\
B^{+}: \quad \varepsilon<w<R \\
A^{-}: \quad w=R e^{i \varphi}, \quad 2 \pi>\varphi>0 \\
B^{-}: \quad R>w>\varepsilon
\end{gathered}
$$

The last term in the r.h.s. of (A.5) gives

$$
\begin{gathered}
{\left[\int_{B^{+}}+\int_{B^{-}}\right] H_{n} d \Omega_{k}=\int_{B}\left(H_{n}^{+} d \Omega_{k}-H_{n}^{-} d \Omega_{k}\right)=2 \pi i \int_{B} z^{n} d \Omega_{k}=} \\
=\left.2 \pi i\left(z^{n} \Omega_{k}-n z^{n-1} \Omega_{k}^{(1)}+\ldots+(-)^{n} n ! \Omega_{k}^{(n)}\right)\right|_{\varepsilon} ^{R}
\end{gathered}
$$


where, similarly to $(3.13)$,

$$
\frac{d^{n} \Omega_{k}^{(n)}}{d z^{n}}=\Omega_{k}, \quad n \geq 0
$$

is introduced.

For the $A$-integrals one can write

$$
\int_{A^{ \pm}} H_{n} d \Omega_{k}=\int_{A^{ \pm}} H_{n}^{(+)}(z) d \Omega_{k} \mp 2 \pi i \operatorname{res}_{P_{ \pm}} z^{k} d H_{n}
$$

where by residue the coefficient at the term $z^{-1}$ is meant. The first term in the r.h.s. of (A.9) can be integrated by parts using (3.3) and (A.8), giving rise to

$$
\begin{gathered}
\int_{A^{+}} H_{n}^{(+)} d \Omega_{k}=\left.\left(H_{n}^{(+)} \Omega_{k}-n H_{n-1}^{(+)} \Omega_{k}^{(1)}+\ldots+(-)^{n} n ! H_{0}^{(+)} \Omega_{k}^{(n)}\right)\right|_{\varepsilon^{-}} ^{\varepsilon^{+}}= \\
=\left.2 \pi i\left(z^{n} \Omega_{k}-n z^{n-1} \Omega_{k}^{(1)}+\ldots+(-)^{n} n ! \Omega_{k}^{(n)}\right)(\varepsilon)\right|_{\operatorname{div}} \\
\int_{A^{-}} H_{n}^{(+)} d \Omega_{k}=\left.\left(H_{n}^{(+)} \Omega_{k}-n H_{n-1}^{(+)} \Omega_{k}^{(1)}+\ldots+(-)^{n} n ! H_{0}^{(+)} \Omega_{k}^{(n)}\right)\right|_{R^{+}} ^{R^{-}}= \\
=-\left.2 \pi i\left(z^{n} \Omega_{k}-n z^{n-1} \Omega_{k}^{(1)}+\ldots+(-)^{n} n ! \Omega_{k}^{(n)}\right)(R)\right|_{\text {div }}
\end{gathered}
$$

where in the r.h.s.'s one gets only the divergent parts of the corresponding expressions.

Altogether (A.4), (A.5), (A.7), (A.9) and (A.10) give rise to

$$
\begin{gathered}
0=\left.\left(z^{n} \Omega_{k}-n z^{n-1} \Omega_{k}^{(1)}+\ldots+(-)^{n} n ! \Omega_{k}^{(n)}\right)\right|_{\varepsilon} ^{R}+ \\
+\left.\left(z^{n} \Omega_{k}-n z^{n-1} \Omega_{k}^{(1)}+\ldots+(-)^{n} n ! \Omega_{k}^{(n)}\right)(\varepsilon)\right|_{\mathrm{div}}- \\
-\left.\left(z^{n} \Omega_{k}-n z^{n-1} \Omega_{k}^{(1)}+\ldots+(-)^{n} n ! \Omega_{k}^{(n)}\right)(R)\right|_{\mathrm{div}}- \\
-\operatorname{res}_{P_{+}} z^{k} d H_{n}+\operatorname{res}_{P_{-}} z^{k} d H_{n}
\end{gathered}
$$

or, using the antisymmetry w.r.t. involution exchanging $P_{+}$and $P_{-}$,

$$
\begin{gathered}
\operatorname{res}_{P_{+}} z^{k} d H_{n}=-\operatorname{res}_{P_{-}} z^{k} d H_{n}= \\
=\left.\left(z^{n} \Omega_{k}-n z^{n-1} \Omega_{k}^{(1)}+\ldots+(-)^{n} n ! \Omega_{k}^{(n)}\right)(\varepsilon)\right|_{\text {const }}= \\
=-\left.\left(z^{n} \Omega_{k}-n z^{n-1} \Omega_{k}^{(1)}+\ldots+(-)^{n} n ! \Omega_{k}^{(n)}\right)(R)\right|_{\text {const }}
\end{gathered}
$$

or

$$
\operatorname{res}_{P_{+}} z^{k} d H_{n}=-\operatorname{res}_{P_{-}} z^{k} d H_{n}=(-)^{n} n !\left[\Omega_{k}^{(n)}\right]_{0}
$$




\section{References}

[1] A. S. Losev, A. Marshakov and N. Nekrasov, "Small instantons, little strings and free fermions," in Ian Kogan memorial volume, M.Shifman, A.Vainshtein and J. Wheater (eds.) From fields to strings: circumnavigating theoretical physics, 581-621 [arXiv:hepth/0302191].

[2] A. Marshakov and N. Nekrasov, JHEP 0701 (2007) 104 [arXiv:hep-th/0612019].

[3] N. Nekrasov, Adv. Theor. Math. Phys. 7 (2004) 831 [arXiv:hep-th/0206161].

[4] I. Krichever, Commun. Pure. Appl. Math. 47 (1992) 437 [arXiv:hep-th/9205110].

[5] T. Eguchi and S. K. Yang, Mod. Phys. Lett. A 9 (1994) 2893 [arXiv:hep-th/9407134].

[6] T. Eguchi, K. Hori and S. K. Yang, Int. J. Mod. Phys. A 10 (1995) 4203 [arXiv:hepth/9503017].

[7] A. Givental, "Gromov-Witten invariants and quantization of quadratic hamiltonians", arXiv:math/0108100.

[8] A. Okounkov and R. Pandharipande, "Gromov-Witten theory, Hurwitz theory, and completed cycles", arXiv:math.AG/0204305; "The equivariant Gromov-Witten theory of $\mathbb{P}^{1}$ ", arXiv:math.AG/0207233.

[9] A. Okounkov and R. Pandharipande, "Virasoro constraints for target curves", arXiv:math/0308097.

[10] G. Carlet, B. Dubrovin and Y. Zhang, "The extended Toda hierarchy", arXiv:nlin/0306060;

B. Dubrovin and Y. Zhang, "Virasoro symmetries of the extended Toda hierarchy", arXiv:math/0308152.

[11] T. Milanov, "Hirota quadratic equations for the extended Toda hierarchy", arXiv:math/0501336; "The equivariant Gromov-Witten theory of $C P^{1}$ and integrable hierarchies", arXiv:math-ph/0508054; "Gromov-Witten theory of $C P^{1}$ and integrable hierarchies", arXiv:math-ph/0605001.

[12] A. Marshakov, "On microscopic origin of integrability in Seiberg-Witten theory," arXiv:0706.2857 [hep-th].

[13] N. Nekrasov and A. Okounkov, "Seiberg-Witten theory and random partitions," arXiv:hepth/0306238.

[14] A. S. Losev, Theor. Math. Phys. 95 (1993) 595 [Teor. Mat. Fiz. 95 (1993) 307] [arXiv:hepth/9211090]. 
[15] B. Logan and L. Shepp, Advances in Math. 26 (1977), no. 2, 206;

S. Kerov and A. Vershik, DAN SSSR, 233,1024(1977), (in Russian).

[16] N. Nekrasov, Nucl. Phys. B531 (1998) 323 [arXiv:hep-th/9609219];

A. Lawrence and N. Nekrasov, Nucl. Phys. B513 (1998) 239 [arXiv:hep-th/9706025];

A. Marshakov and A. Mironov, Nucl. Phys. B 518 (1998) 59 [arXiv:hep-th/9711156];

H. Braden, A. Marshakov, A. Mironov and A. Morozov, Phys. Lett. B 448 (1999) 195 [arXiv:hep-th/9812078]; Nucl. Phys. B 558 (1999) 371 [arXiv:hep-th/9902205].

[17] T. Maeda, T. Nakatsu, K. Takasaki and T. Tamakoshi, Nucl. Phys. B 715 (2005) 275 [arXiv:hep-th/0412329];

T. Nakatsu and K. Takasaki, "Melting crystal, quantum torus and Toda hierarchy," arXiv:0710.5339 [hep-th]. 